\title{
Interpolation of weakly compact operators
}

\author{
By
}

Lech Maligranda and Alexi Quevedo

Let $\bar{A}=\left(A_{0}, A_{1}\right)$ be a Banach pair, i.e., $A_{0}$ and $A_{1}$ are Banach spaces continuously imbedded into a Hausdorff topological vector space. Define, as usual, the spaces $\Delta(\bar{A})=A_{0} \cap A_{1}$ and $\Sigma(\bar{A})=A_{0}+A_{1}$ with the norms $\|a\|_{A}=\max \left(\|a\|_{A_{0}},\|a\|_{A_{1}}\right)$ and $\|a\|_{\Sigma}=K(1, a)$, where for $t>0$,

$$
K(t, a)=\inf \left\{\left\|a_{0}\right\|_{A_{0}}+t\left\|a_{1}\right\|_{A_{1}}: a=a_{0}+a_{1}, a_{0} \in A_{0}, a_{1} \in A_{1}\right\} .
$$

For $0<\theta<1$ and $1 \leqq p<\infty$, the Lions-Peetre interpolation space $\bar{A}_{\theta, p}=\left(A_{0}, A_{1}\right)_{\theta, p}$ is the space of all $a \in \Sigma(\bar{A})$ for which the norm

$$
\|a\|_{\theta, p}=\left(\sum_{n \in \mathbb{Z}}\left(2^{-\theta n} K\left(2^{n}, a\right)\right)^{p}\right)^{1 / p}
$$

is finite (cf. [2]). Let us note that $\mu_{n}(a) \leqq K\left(2^{n}, a\right) \leqq 2 \mu_{n}(a)$, where $\mu_{n}$ is the Minkowski functional of the set $V_{n}=B_{A_{0}}+2^{-n} B_{A_{1}}\left(B_{A_{i}}\right.$ is the unit ball of $A_{i}, i=0$, 1), i.e., $\mu_{n}(a)$ $=\inf \left\{\alpha>0: a \in \alpha V_{n}\right\}$. Hence $\bar{A}_{\theta, p}$ consists of those $a \in \Sigma(\bar{A})$ for which the norm

$$
\|a\|_{\theta, p}^{1}=\left(\sum_{n \in \mathbb{Z}}\left(2^{-\theta n} \mu_{n}(a)\right)^{p}\right)^{1 / p}
$$

is finite. If in the above definitions sums are only over natural $n$ then we have the definition of the space $\bar{A}_{\theta, p}^{+}$. Always $\bar{A}_{\theta, p} \subset A_{\theta, p}^{+}$and if $A_{\theta} \subset A_{1}$, then $\bar{A}_{\theta, p}=\bar{A}_{\theta, p}^{+}$.

Now let $\bar{A}=\left(A_{0}, A_{1}\right)$ and $\bar{B}=\left(B_{0}, B_{1}\right)$ be two Banach pairs. We say that a linear operator $T$ is bounded from the pair $\bar{A}$ into the pair $\bar{B}$, and write $T: \bar{A} \rightarrow \bar{B}$, if $T: \Sigma(\bar{A}) \rightarrow \Sigma(\bar{B})$ is a bounded linear operator and the restriction of $T$ to the space $A_{i}$ is a bounded operator from $A_{i}$ into $B_{i}, i=0,1$.

From the construction of the Lions-Peetre spaces we have that if $T: \bar{A} \rightarrow \bar{B}$, then $T: \bar{A}_{\theta, p} \rightarrow \bar{B}_{\theta, p}$ is bounded and

$$
\|T\|_{\bar{A}_{\theta, p} \rightarrow \bar{B}_{\theta, p}} \leqq\|T\|_{A_{0} \rightarrow B_{0}}^{1-\theta}\|T\|_{A_{1} \rightarrow B_{1}}^{\theta} .
$$

The following result can be regarded as a generalization of the theorem of Beauzamy [1] about the reflexivity of $\bar{A}_{\theta, p}$. Moreover, our proof will be simpler.

Theorem 1. Let $1<p<\infty$ and let $T: \bar{A} \rightarrow \bar{B}$. Then $T: \bar{A}_{\theta, p} \rightarrow \bar{B}_{\theta, p}$ is weakly compact if and only if $T: \Delta(\bar{A}) \rightarrow \Sigma(\bar{B})$ is weakly compact. 
The proof will follow from two Propositions, which themselves are of independent interest. Before we do this let us recall the DFJP construction. Let $X$ be a Banach space and let $W$ be a convex symmetric, norm bounded subset of $X$. For each $n$ put $U_{n}=W+2^{-n} B_{X}$ and denote by $j_{n}$ the Minkowski functional of $U_{n}$. For $0<\theta<1$ and $1<p<\infty$ set $Y=Y_{\theta, p}=\left\{x \in X:\|x\|=\left(\sum_{n \in \mathbb{N}}\left(2^{-\theta n} j_{n}(x)\right)^{p}\right)^{1 / p}<\infty\right\}$, and let $J: Y \rightarrow X$ denote the natural inclusion. Then (cf. [4], where in fact the space $Y_{1 / 2,2}$ is considered:

1. $Y$ is a Banach space and $J$ is continuous;

2. $W$ is a subset of $J\left(B_{Y}\right)$;

3. $J^{* *}: Y^{* *} \rightarrow X^{* *}$ is one to one and $J^{* *-1}(X)=Y$;

4. The $\sigma\left(X^{* *}, X^{*}\right)$-closure of $J\left(B_{Y}\right)$ is $J^{* *}\left(B_{Y^{* *}}\right)$;

5. $Y$ is reflexive if and only if $W$ is a relatively weakly compact subset of $X$.

We refer to $Y$ as the space of Davis, Figiel, Johnson and Pelczynski or DFJP space in short. Another important property associated with the space $Y$, needed for our work, is described in the next lemma.

Lemma 2. Let $T$ be a continuous operator from $X$ into any Banach space $Z$. Then $S=T J: Y \rightarrow Z$ is weakly compact if and only if $T(W)$ is relatively weakly compact in $Z$.

Proof. Since $W \subset J\left(B_{Y}\right)$ it follows that $T(W) \subset T J\left(B_{Y}\right)=S\left(B_{Y}\right)$ and if $S$ is weakly compact then $T(W)$ is relatively weakly compact.

Suppose now that $T(W)$ is relatively weakly compact in $Z$.

First of all, the $\sigma\left(Z^{* *}, Z^{*}\right)$-closure of $S\left(B_{Y}\right)$ is $S^{* *}\left(B_{Y^{*}}\right)$. In fact,

$$
\begin{aligned}
\overline{S\left(B_{Y}\right)} & =\overline{S^{* *}\left(B_{Y}\right)} \subset \overline{S^{* *}\left(B_{\left.Y^{\star *}\right)}\right.}=S^{* *}\left(B_{Y^{\star *}}\right) \subset T^{* *}\left(\overline{\left.J\left(B_{Y}\right)^{\sigma\left(X^{* *}, X^{*}\right)}\right)}\right. \\
& =T^{* *}\left(\overline{\left.J^{* *}\left(B_{Y}\right)^{\sigma\left(X^{* *}, X^{\star}\right)}\right)} \quad\left[T^{* *} \text { is } \omega^{*} \text { continuous }\right]\right. \\
& \subset \overline{S^{* *}\left(B_{Y}\right)}=\overline{S\left(B_{Y}\right)} .
\end{aligned}
$$

Now, we have $J\left(B_{Y}\right) \subset 2^{\theta n} U_{n}=2^{\theta n} W+2^{(\theta-1) n} B_{X}$ and so

$$
\begin{aligned}
& T J\left(B_{Y}\right) \subset T\left(2^{\theta n} U_{n}\right)=2^{\theta n} T(W)+2^{(\theta-1) n} T\left(B_{X}\right) \text { [from the continuity of } T \text { ] } \\
& \subset 2^{\theta n} T(W)+2^{(\theta-1) n} M B_{Z}
\end{aligned}
$$

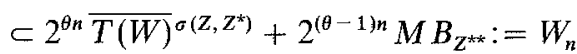

for every $n$. Since $\overline{T(W)}^{\sigma\left(Z, Z^{*}\right)}$ is weakly compact then it is also compact in the $\sigma\left(Z^{* *}, Z^{*}\right)$ topology and the sets $W_{n}$ are $\sigma\left(Z^{* *}, Z^{*}\right)$ closed and contain $S\left(B_{Y}\right)$, therefore they contain $S^{* *}\left(B_{Y^{*}}\right)$. Thus

$$
\begin{aligned}
S^{* *}\left(B_{Y^{\star \star}}\right) & \subset \bigcap_{n}\left(2^{\theta n} \overline{T(W)}+2^{(\theta-1) n} M B_{Z^{\star \star}}\right) \\
& \subset \bigcap_{n}\left(Z+2^{(\theta-1) n} M B_{Z^{\star \star}}\right)=\bar{Z}^{Z^{\star \star}}=Z .
\end{aligned}
$$

Hence $S^{* *}\left(Y^{* *}\right) \subset Z$ and so $S=T J$ is weakly compact. 
As a consequence of the Lemma 2 we have

Proposition 3. Let $T: \Sigma(\bar{A}) \rightarrow Z$ be a continuous operator. The following are equivalent:

(a) $T: \Delta(\bar{A}) \rightarrow Z$ is weakly compact.

(b) For all $0<\theta<1$ and all $1<p<\infty, T:(\Delta(\bar{A}), \Sigma(\bar{A}))_{\theta, p} \rightarrow Z$ is weakly compact.

(c) For all $0<\theta<1$ and all $1<p<\infty, T: \bar{A}_{\theta, p} \rightarrow Z$ is weakly compact.

P r o of. (a) $\Rightarrow$ (b). The interpolation space $(\Delta(\bar{A}), \Sigma(\bar{A}))_{\theta, p}$ is a DFJP space constructed from $X=\Sigma(\bar{A})$ and $W=I\left(B_{\Delta(\bar{A})}\right)$, where $I$ is the inclusion $I: \Delta(\bar{A}) \rightarrow \Sigma(\bar{A})$. Using Lemma 2 we have the above implication.

(b) $\Rightarrow$ (c). The interpolation space $\bar{A}_{\theta, p}$ is not, in general, a DFJP space but such as is proved in [9] they are imbedded into $(\Delta(\bar{A}), \Sigma(\bar{A}))_{\theta, p}$, i.e., if $1 / 2 \leqq \theta<1$ then

$$
(\Delta(\bar{A}), \Sigma(\bar{A}))_{\theta, p}=(\Sigma(\bar{A}), \Delta(\bar{A}))_{1-\theta, p}=\bar{A}_{\theta, p}+\bar{A}_{1-\theta, p}
$$

and so $\bar{A}_{\theta, p}$ and $\bar{A}_{1-\theta, p}$ are in $(\Delta(\bar{A}), \Sigma(\bar{A}))_{\theta, p}$.

That (c) implies (a) is obvious, because $\bar{A}_{\theta, p} \supset \Delta(\bar{A})$.

$\mathrm{R} \mathrm{e} \mathrm{m} \mathrm{a} \mathrm{rk} \mathrm{4.} \mathrm{Lemma} 2$ and Proposition 3 are also true for compact, weakly precompact (= Rosenthal) and Banach-Saks operators. Moreover the equivalence between (a) and (b) is true even for surjective closed operator ideals (see [6], Prop. 1.7).

Proposition 5. Let $1<p<\infty$ and let $T: Z \rightarrow \bar{B}_{\theta, p}$ be a continuous operator. Then $T$ is weakly compact if and only if $T: Z \rightarrow \Sigma(\bar{B})$ is weakly compact.

P r o of. Since the necessity is clear, we will only prove sufficiency. First, we prove that if $1<p<\infty$ then the imbedding $J: \bar{B}_{\theta, p} \rightarrow \Sigma(\bar{B})$ is a Tauberian operator, i.e., $J^{* *} b^{* *} \in \Sigma(\bar{B})$ implies $b^{* *} \in \bar{B}_{\theta, p}$ (see also [1], p. 28).

Let $B=\left(\sum_{n \in \mathbb{Z}} \oplus \Sigma_{n}\right)_{p}$, where $\Sigma_{n}=\Sigma(\bar{B})$ with the norm $\|b\|_{n}=2^{-\theta n} K\left(2^{n}, b\right)$. Then $J=P R$, where $R: \bar{B}_{\theta, p} \rightarrow B$ is the into isometry given by $R(b)=(\ldots, b, b, b, \ldots)$, $P: B \rightarrow \Sigma_{0}=\Sigma(\bar{B})$ is the zero coordinate projection. Then $B^{* *}=\left(\sum_{n \in \mathbb{Z}} \oplus \Sigma_{n}^{* *}\right)_{p}$ and $P^{* *}: B^{* *} \rightarrow \Sigma_{0}^{* *}$ is again the zero coordinate projection. Suppose that $J^{* *} b^{* *} \in \Sigma(\bar{B})$. Then $P^{* *} R^{* *} b^{* *} \in \Sigma_{0}$ and $R^{* *} b^{* *}=\left(\ldots, d_{-1}^{* *}, d, d_{1}^{* *}, d_{2}^{* *}, \ldots\right)$ where $d \in \Sigma_{0}$. There exists a net $\left(b_{\alpha}\right)$ such that $\left(R b_{\alpha}\right)$ converges in the weak* topology to $R^{* *} b^{* *}$. Since every coordinate of $\left(R b_{\alpha}\right)$ is equal and each $\sum_{n}^{* *}$ is isomorphic to $\sum(\bar{B})^{* *}, R^{* *} b^{* *}$ $=(\ldots, d, d, d, \ldots)=R d \in B$. Since $R^{* *}$ is one to one, $b^{* *}=d \in \bar{B}_{\theta, p}$.

Now if $J T\left(B_{Z}\right)$ is relatively weakly compact in $\Sigma(\bar{B})$ then the weak*-closure of $J T\left(B_{Z}\right)$ in $\Sigma(\bar{B})^{* *}$ is contained in $\Sigma(\bar{B})$, i.e., $\widetilde{T\left(B_{Z}\right)} \subset \Sigma(\bar{B})$. Since $J^{* *}$ is weak ${ }^{*}$-to-weak* continuous,

$$
J * *\left(\widetilde{T B_{Z}}\right) \subset \widetilde{J T B_{Z}} \subset \Sigma(\bar{B}) .
$$

Since $J$ is Tauberian, $\widetilde{T B_{Z}} \subset \bar{B}_{\theta, p}$. But $T B_{Z}$ is bounded and so $T B_{Z}$ is relatively weakly compact in $\bar{B}_{\theta, p}$.

The last property characterizes Tauberian operators (cf. [7], Th. 3.2 and [10], Th. 1.4). 
Proof of The orem 1 . If $T: \bar{A} \rightarrow \bar{B}$ is such that $T: \Delta(\bar{A}) \rightarrow \Sigma(\bar{B})$ is weakly compact, then $T: \bar{A}_{\theta, p} \rightarrow \bar{B}_{\theta, p}$ is bounded and by Proposition $3 T: \bar{A}_{\theta, p} \rightarrow \Sigma(\bar{B})$ is weakly compact. Then by Proposition 5T: $\bar{A}_{\theta, p} \rightarrow \bar{B}_{\theta, p}$ is weakly compact.

The converse implication is obvious because $\bar{A}_{\theta, p} \supset \Delta(\bar{A})$ and $\bar{B}_{\theta, p} \subset \Sigma(\bar{B})$.

Remarks 6. Proposition 5 and Theorem 1 are also true for weakly precompact operators. Theorem 1 is true even for properties which are preserved by Proposition 3 and Tauberian operators.

7. The techniques used above also work for the (more general) method of interpolation with a function parameter. We refer to [5] for details of this method.

If in our Theorem the operator is taken to be the identity, then we have the result of Beauzamy. Namely, $\bar{A}_{\theta, p}(0<\theta<1,1<p<\infty)$ is reflexive if and only if the imbedding $I: \Delta(\bar{A}) \rightarrow \Sigma(\bar{A})$ is weakly compact.

Another proof of this result is the following:

If the imbedding $I$ is weakly compact, then the DFJP space $(\Delta(\bar{A}), \Sigma(\bar{A}))_{1 / 2, p}$ is reflexive (see [4]). But from [9] we have that $(\Delta(\bar{A}), \Sigma(\bar{A}))_{1 / 2, p}=\bar{A}_{1 / 2, p}$ and from the reiteration theorem for the complex interpolation space (see [2], Th. 4.7.2)

$$
\bar{A}_{\theta, p}=\left[\bar{A}_{\theta_{0}, p}, \bar{A}_{1 / 2, p}\right]_{\theta_{1}}
$$

for some $\theta_{0}$ and $\theta_{1}$ from $(0,1)$. Now, using the above and Calderón's result (if either $A_{0}$ or $A_{1}$ is reflexive space then the complex interpolation space $\left[A_{0}, A_{1}\right]_{\theta}$ is also reflexive-see [3], Th. 12.2) we have that $\bar{A}_{\theta, p}$ is reflexive.

Let us note that in the nontrivial case, i.e. when the imbedding $I$ is nonclosed, the space $\bar{A}_{\theta, 1}$ is nonreflexive. Indeed, by Levy's [8] result it contains isomorphic copy of $\ell^{1}$.

\section{References}

[1] B. BeauZAmy, Espaces d'interpolation réels: Topologie et géométrie. LNM 666, Berlin-Heidelberg-New York 1978.

[2] J. BERGH and J. LöFSTROM, Interpolation spaces. An introduction. Berlin-Heidelberg-New York 1976.

[3] A. P. CALDERón, Intermediate spaces and interpolation, the complex method. Studia Math. 24, $133-190$ (1964).

[4] W. Davis, T. Figiel, W. Johnson and A. Pelczynski, Factoring weakly compact operators. J. Funct. Anal. 17, 311-327 (1974).

[5] J. Gustavsson, A function parameter in connection with interpolation of Banach spaces. Math. Scand. 42, 289-305 (1978).

[6] S. Heinrich, Closed operator ideals and interpolation. J. Funct. Anal. 35, 397-411 (1980).

[7] N. Kalton and A. WILANSKy, Tauberian operators on Banach spaces. Proc. Amer. Math. Soc. 57, 251-255 (1976).

[8] M. Levy, L'espace d'interpolation réel $\left(A_{0}, A_{1}\right)_{\theta, p}$ contient $\ell_{p}$. C.R. Acad. Sci. Paris 289, $675-677$ (1979). 
[9] L. Maligranda, Interpolation between sum and intersection of Banach spaces. J. Approx. Theory 47, 42-53 (1986).

[10] R. NEIDINGER, Factoring operators through hereditarily- $\ell^{p}$ spaces. LNM 1166, 116-128, Berlin-Heidelberg-New York 1985.

Eingegangen am 2.2.1989

Anschriften der Autoren:

Lech Maligranda

Departamento de Matemáticas

IVIC

Apartado 21827

Caracas 1020-A

venezuela

\author{
Alexi Quevedo \\ Departamento de Matemáticas \\ Facultad de Ciencias \\ Universidad Central de Venezuela \\ Apartado 20513 \\ Caracas 1020-A \\ Venezuela
}

